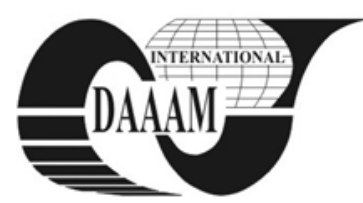

Annals of DAAAM for 2011 \& Proceedings of the 22nd International DAAAM Symposium, Volume 22, No. 1, ISSN 1726-9679 ISBN 978-3-901509-83-4, Editor B. Katalinic, Published by DAAAM International, Vienna, Austria, EU, 2011 Make Harmony between Technology and Nature, and Your Mind will Fly Free as a Bird Annals \& Proceedings of DAAAM International 2011

\title{
IMPLEMENTATION OF ROBOT OFFLINE PROGRAMMING
}

\author{
BOZEK, P[avol] \& CHMELIKOVA, G[abriela]
}

\begin{abstract}
The paper deals with the practical offline programming of a virtual robot. Offline programming is the best way how to maximalize the return of investments of robotic workplaces. Programming of a robot on PC (Našćák \& Šurianský, 2000) without the need of production stop is enabled by software for simulation and offline. It also enables to prepare robot programmes in advance, what increases overall productivity.
\end{abstract}

Keywords: virtual environment, robot, simulation, learning

\section{INTRODUCTION}

The aforementioned system provides tools leading to a profitability increase of a robotic system in the way that tasks such as training, programming and optimalization without the intervention necessity of a production process are allowed to carry out. It brings a lot of advantages, for example hazard decreasing, faster launching of operation, faster changes of settings and production increasing.

\section{PRACTICAL IMPLEMENTATION PROGRAMMING OFFLINE}

The aim of object management in a virtual scene is a fully operational virtual industrial robot managed in the same way as in practice. For the simplicity of an operator service as well as simplicity of an access to single functions in a user regime it is suitable for the purposes of teaching a management and programming $\mathrm{NC}$ devices system in various levels of service personnel educating (Cupec et al, 2003).

\subsection{Mathematical description of a robot}

For successful solving the project, as a start, it is necessary to sort out a mathematical description of a robot by which we determine relations among the number of performed which we determine relations among the number of performed

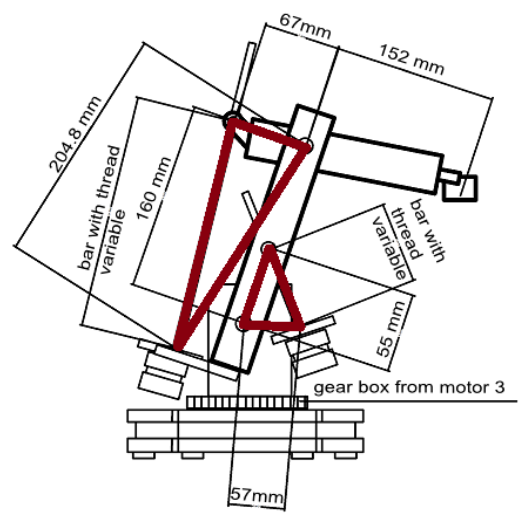

Fig. 1. Laboratory DP01 angulatory robot with determined triangles necessary for the calculation of motor steps

\subsection{Mathematical application of the law of cosines}

Motor steps of and angles of arm slewing or coordinates of the effector as it is in figure 1.

It is possible to use the following mathematical reflections for determining the number of single motor steps to be carried out for shifting the magnetic gripper from point $[\mathrm{X} 0, \mathrm{Y} 0, \mathrm{Z} 0]$ to point $[\mathrm{X} 1, \mathrm{Y} 1, \mathrm{Z} 1]$ or vice-versa.For describing a general triangle from mathematics it is known the law of cosines Refer to "(1),", where "a", "b", "c" are the sides of a triangle and the angle alpha is the angle that „,

$$
a^{2}=b^{2}+c^{2}-2 \cdot b \cdot c \cdot \cos (\text { alpha })
$$

The aforementioned known formulation serves for the calculation of slewing of three driving units for a change of the magnetic gripper position in space and vice-versa.

\subsection{The determining of electromagnetic effector coordinates}

The general triangle constructed from a main arm, auxiliary arm (including gripper) and imaginary line from anchoring of the main arm to the gripper forms the basis for the calculation of the gripper position in space.

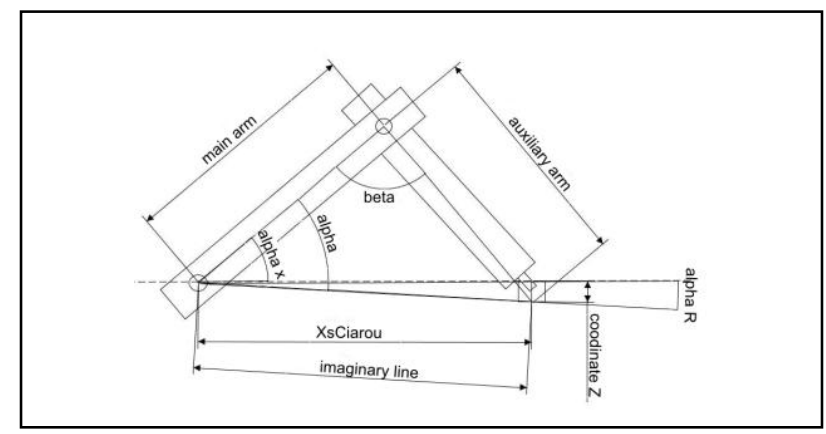

Fig. 2. Basic calculating triangle of a vertical plane

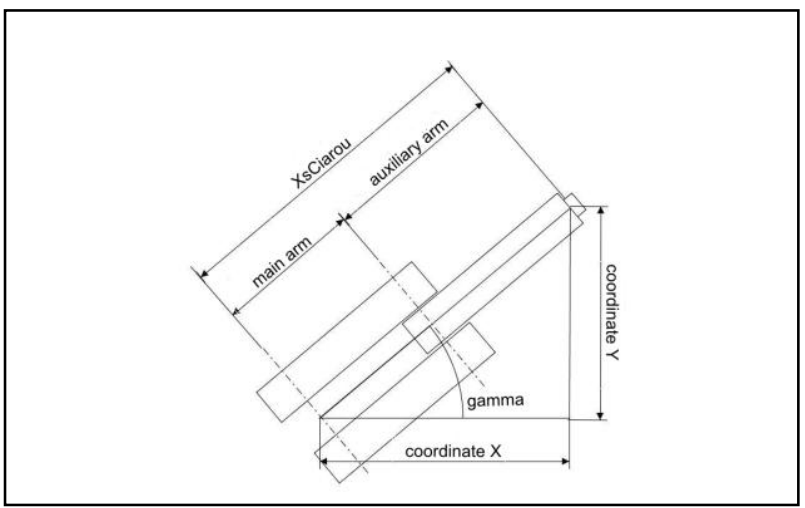

Fig. 3. Basic calculating triangle of a horizontal plane

The angle beta (angle between main and auxiliary arm) and angle alpha $\mathrm{R}$ (angle between main arm and imaginary line) are 
important angles in this triangle. The triangle is shown in figure 2,3 . On the basis of these reflections we might determine the angles of single robot arms from the magnetic gripper coordinates is space and vice-versa (Karavaev et al, 2008).

2.4 Determining coordinates of the magnetic gripper from the assigned angles

$$
\begin{aligned}
& r()= \\
& \sqrt{\text { rameno }^{2}+\text { rameno }^{2}-2 \text {.rameno2.ramenol.cos(betaZadane) }} \\
& \text { alfaR }()=\text { alfaXZadane }- \\
& \operatorname{ArcCos}\left(\left(\text { rameno }^{2}-r()^{2}-\text { rameno }^{2}\right) / 2 . \text { ramenol } r()\right) \\
& \text { _ } z(\mathrm{O})=r() \cdot \sin (\operatorname{alfaR}()) \\
& \text { _ Xs Ciarov }()=r() \cdot \cos (\operatorname{alfaR}()) \\
& \text { _y }()=X s \text { Ciarov1 }() \cdot \cos (\text { gamaZadane }) \\
& \text { _ } x()=\text { XsCiarov1().sin( gamaZadane })
\end{aligned}
$$

\section{APPLICATION OFFLINE PROGRAMMING OF A ROBOT IN VIRTUAL ENVIRONMENT}

A solutionist has to suggest and optimalize a robotic workplace before he starts to form the robot programme. This can be done in real environment and consequently he can form a copy of suggested workplace in virtual environment or he can use virtual reality environment of VIRTUAL ROBOT application to propose a robotic cell. For the proposal needs some models of objects e.g. various palettes, conveyors, stands and of course DP01 robot itself has already been prepared.

\subsection{Application possibilities in a virtual scene}

The aim of object management in a virtual scene is a fully operational virtual production system with an industrial robot managed in the same way as in practice. For the simplicity of an operator service as well as the simplicity of an approach to single functions in a user regime, the virtual industrial robot is exceptionally suitable for the purposes of teaching a management and programming $\mathrm{NC}$ devices system in different levels of educating the service personnel (Śtollmann, 2006). Next, it is possible to use a virtual automatic system of a technological workplace in laboratory conditions to train operators and programmers of uninterrupted operations. Apart from this, automatic production systems known as the periphery of the industrial robot will be possibly and simply incorporated into simulation of a virtual robotic complex. All the arms of the angulatory robot carry out rotational movement with six degrees of freeedom. Kinematics of such a robot has open kinematic chain. There are basic components of the angulatory robot:

Base - is a robot part that is connected firmly with the ground

$>$ Arms - are fixed robot parts

$>$ Joints - are robot parts that allow free or managed movement of two arms that are connected by a joint

$>$ Gripper - robot end effector that serves for holding, object manipuation or the assembling of next devices, e.g. spray or welding heads.

$>$ Kinematic pair - is a pair of arms connected with a joint

$>$ Kinematic chain - is a set of arms connected with joints

The kinematic chain can be represented by a graph where joints form hitches and arms form edges of the graph. The axes of rotation pass through points which are considered to be typical points in kinematics of the angulatory robot. Tough the axis of rotation is not unambiguously determined around a point, it is necessary to determine the axis of a coordinate system that is parallel with this axis of rotation. By the perception of coordinate systems via the optics of view Open
GL it is shifting the coordinate system towards an identified point as the characteristic one.

\section{ELECTRONICS OF DP01 ROBOT}

The electronics managing DP01 robot consists of these constructional parts:

1. Atmel AT 89S2051 Microcontroller.

2. Expanding of 8-bit signal from the microcontroller to 16bit necessary to manage DP01 laboratory robot by means of two SN74LS373integrated circuits.

3. The stepper motor driver (amplifier) transforming low memory signals from a control circuit to current signals, necessary to drive single rolling of the stepper motor is formed mainly by ULN2803 integrated circuits, from which a current signal is amplified by BD140 transistors.

The export from virtual environment into the real one is carried out by means of the computer serial port of through which the robot programme formed offline by means of virtual robot application we transfer into the memory of ATMEL microcontroller (Šurianský \& Frank, 2006). Then, it is carried out by means of its programme. It means that the microcontroller sends signals into stepper motor drivers that activate single motors as it is mentioned in the carried out programme, eventually it turns on or turns off the electromagnetic gripper.

\section{CONCLUSION}

A described laboratory is set on a virtual control system of an exact copy of real software that manages a robot model. Therefore it permits very realistic simulations with the use of real robotic programmes and configuration files identical with those that are used in production. The described results are suitable not only for simulation but also for student training.

\section{ACKNOWLEDGEMENTS}

The contribution was elaborated within the research project KEGA project No. 3-7285-09 Contents Integration and Design of University Textbook "Specialised Robotic Systems" in Print and Interactive Modules for University of Technology in Zvolen, Trenčín University and Slovak University of Technology in Bratislava.

\section{REFERENCES}

Cupec, R. ; Lorch, O. \& Schmidt, G. (2003) Vision-Guided Humanoid Walking. Concepts and Experiments, Proceedings of the 12th International Workshop on RAAD, Cassino, Italija, Svibanj

Karavaev, J.L. ; Lekomtsev, P. \& Abramov, A.I. (2008) Comparative analysis of the force parameters for drilling plastic and brittle materials. - Bulletin IzhSTU № 1, Izhevsk, pp. 10-15, ISSN 1813-7903

Nascak, L, \& Suriansky, J. (2000) Measuring systems with computers. Scientific studies 7/2000A. Technical University in Zvolen, pp. 79, ISBN 80228-1017-7

Štollmann, V. (2006) Derivation of relation for the calculation of stepping barriers depending on the radius of the wheel. In: AFF, Zvolen, Slovakia, ISSN 0231-5785, pp. 377-382.

Surianský, J. \& Frank, T. (2006) Visualisation of magnetic fields generated by Helmholtz coils. In Elektro. International konfernce, 2006, Technical university Žilina, pp. 326, ISSN 1336-1376 